\title{
Flexoelectric Coefficients for Model Pear Shaped Molecules from Monte Carlo Simulations
}

\author{
JOACHIM STELZER, ROBERTO BERARDI and \\ CLAUDIO ZANNONI
}
Dipartimento di Chimica Fisica ed Inorganica, Università degli Studi di Bologna, Viale del Risorgimento 4, I-40136 Bologna, Italy

The flexoelectric effect for pear-shaped molecules was studied within NPT Monte Carlo simulations. The asymmetric molecules were modelled by a heterogenic two-site pair potential consisting of rigidly connected Gay-Berne and Lennard-Jones particles, thus giving rise to a steric dipolar moment. The simulation data for order parameters and pair correlation functions were used within a density functional approach to flexoelectric deformations. Due to the locally antisymmetric arrangement of the molecular steric dipoles, the flexoelectric effect turns out to be rather small. In particular, the bend flexoelectric constant is almost vanishing.

Keywords: computer simulations; microscopic theories

\section{INTRODUCTION}

The flexoelectric effect in liquid crystals as an orientational analogue to piezoelectricity in solid crystals was first predicted by Meyer ${ }^{[t]}$. If the molecules possess an asymmetry of shape there is a linear coupling between an elastic splay or bend deformation of the director 
field $\mathbf{n}(\mathbf{r})$ and a macroscopic polarization $P$,

$$
\mathbf{P}=e_{11} \mathbf{n} \operatorname{div} \mathbf{n}+e_{33} \mathbf{n} \times \operatorname{curl} \mathbf{n},
$$

where the coupling constants $e_{11}$ and $e_{33}$ are called flexoelectric coefficients. They measure the strength of the respective flexoelectric distortions. Flexoelectricity is of fundamental interest, since its relation to molecular structure is not yet understood. There are two prototypes of molecular asymmetry, namely, pear-like and bananalike, which, according to Meyer ${ }^{[1]}$ should exhibit pure splay or bend flexoelectricity, respectively. In the present study we concentrate on the former case of pear-shaped molecules. A simple molecular model, built from a Gay-Berne and a Lennard-Jones center, is introduced and then employed within Monte Carlo computer simulations. Such calculations provide structural properties, like order parameters and pair correlations. These are taken as input quantities in a density functional theory of flexoelectricity ${ }^{[2]}$, in order to obtain estimates for the flexoelectric coefficients.

\section{MICROSCOPIC EXPRESSIONS FOR FLEXOELECTRIC COEFFICIENTS}

A density functional approach to flexoelectricity has been established by Straley ${ }^{[2]}$. Within this theory the polarization $\mathbf{P}$ is obtained as an average of the molecular steric dipoles, of strength $p$, whose weight is the orientational distribution function (ODF), $f\left(\mathbf{u}_{i}\right)$, of the molecular axes $\mathbf{u}_{i}$,

$$
\mathbf{P}=\rho p \operatorname{du} \mathbf{u}_{i} f\left(\mathbf{u}_{i}\right) \delta f\left(\mathbf{u}_{i}\right) \mathbf{u}_{i} .
$$

The flexoelectric deformation acts as a small perturbation to the equilibrium feature of the ODF. This perturbation can be expressed by the direct pair correlation function (DPCF), $c\left(\mathbf{r}_{i j}, \mathbf{u}_{i}, \mathbf{u}_{j}\right)$, which depends on the axes $\mathbf{u}_{i}$ and $\mathbf{u}_{j}$ and on the separation vector $\mathbf{r}_{i j}$ of a pair of molecules,

$$
\delta f\left(\mathbf{u}_{i}\right)=\rho \int \mathrm{d} \mathbf{r}_{i j} \mathrm{~d} \mathbf{u}_{j} c\left(\mathbf{r}_{i j}, \mathbf{u}_{i}, \mathbf{u}_{j}\right)\left(\mathbf{r}_{i j} \cdot \boldsymbol{\nabla}\right) f\left(\mathbf{u}_{j}\right) .
$$

Next, we assume that the ODF is locally at equilibrium, which means that the individual molecular axes follow the director field, and hence 
$f\left(\mathbf{u}_{i}\right)=f\left(\mathbf{u}_{i} \cdot \mathbf{n}(\mathbf{r})\right)$. At this stage director gradients enter the expression (3). After inserting backward (3) into (2), by a comparison between the microscopic form (2) of the flexoelectric polarization to its macroscopic version (1) we obtain general expressions for the flexoelectric coefficients,

$$
\begin{aligned}
& e_{11}=\rho^{2} p \int \mathrm{d} \mathbf{r}_{i j} \mathrm{~d} \mathbf{u}_{i} \mathrm{~d} \mathbf{u}_{j} f\left(\mathbf{u}_{i}\right) u_{i, z} f^{\prime}\left(\mathbf{u}_{j}\right) u_{j, x} r_{i j, x} c\left(\mathbf{r}_{i j}, \mathbf{u}_{i}, \mathbf{u}_{j}\right)(4) \\
& e_{33}=\rho^{2} p \int \mathrm{d} \mathbf{r}_{i j} \mathrm{~d} \mathbf{u}_{i} \mathrm{~d} \mathbf{u}_{j} f\left(\mathbf{u}_{i}\right) u_{i, x} f^{\prime}\left(\mathbf{u}_{j}\right) u_{j, x} r_{i j, z} c\left(\mathbf{r}_{i j}, \mathbf{u}_{i}, \mathbf{u}_{j}\right) .(5)
\end{aligned}
$$

Here $\rho$ means density and $p$ stands for the steric dipole. $f^{\prime}$ denotes the derivative of $f$ with respect to its argument.

The solid angle integrals in (4) and (5) can be performed by means of an expansion into spherical harmonics ${ }^{[3]}$. The flexoelectric coefficients then depend on generalized scalar order parameters $\left\langle P_{L}\right\rangle$ and on radial integrals of the third moments of the DPCF expansion coefficients,

$$
I_{L_{1}, L_{2}, L}^{3}=\int \mathrm{d} r_{i j} r_{i j}^{3} c_{L_{1}, L_{2}, L}\left(r_{i j}\right) .
$$

Importantly, due to the shape asymmetry of the molecules both even and odd quantum numbers $L_{1}, L_{2}, L$ enter the expressions. Therefore, up to second order the flexoelectric coefficients read

$$
\begin{aligned}
e_{11}= & \sqrt{2 \pi} \rho^{2} p\left[\left(\sqrt{2} I_{0,1,1}^{3}+\frac{2}{5} I_{2,1,1}^{3}\right)\left\langle P_{1}\right\rangle^{2}\right. \\
& \left.-I_{1,2,1}^{3}\left(\left\langle P_{2}\right\rangle+2\left\langle P_{2}\right\rangle^{2}\right)\right]+\ldots \\
e_{33}= & \sqrt{2 \pi} \rho^{2} p\left[-\frac{3}{5} I_{2,1,1}^{3}\left(P_{1}\right\rangle^{2}+I_{1,2,1}^{3}\left(-\left\langle P_{2}\right\rangle+\left\langle P_{2}\right\rangle^{2}\right)\right]+\ldots(8)
\end{aligned}
$$

\section{MONTE CARLO SIMULATIONS}

In order to calculate the flexoelectric coefficients from (7) and (8) we need data for the generalized scalar order parameters $\left\langle P_{L}\right\rangle$ and for the DPCF. We obtained these quantities from Monte Carlo simulations for a model system of pear-shaped molecules. The Monte Carlo simulation was thus used as a source for input data for the evaluation of the density functional expressions (7) and (8). In our model each molecule consists of two overlapping ellipsoidal and spherical sites 
which are rigidly connected. The spherical site, with radius $r_{\mathbf{B}}$, was placed on the axis of the ellipsoidal site at a distance $d$ such that the ends of the two sites coincided. In this way we achieved an asymmetry of the molecular shape which resembles a pear. The resulting steric dipole is due to the volume of the spherical site and the site-site distance, yielding $p=\frac{4 \pi}{3} r_{\mathrm{B}} d$. The interaction energy between the spherical sites was given by the well-known 12-6 Lennard-Jones potential, whereas the ellipsoidal site interaction was modelled by the uniaxial Gay-Berne potential ${ }^{[4]}$, with parametrization $\kappa=3, \kappa^{\prime}=5$, $\mu=1, \nu=3$. In addition, the interaction between heterogenic sites was calculated from a generalized pair potential ${ }^{[5]}$. By varying the radius $r_{\mathrm{B}}$ of the Lennard-Jones site we were able to change the steric dipole moments $p$ of the molecules. Three different models $\left(p^{*}=0.814,0.662,0.524\right)$ were investigated in detail, by performing Monte Carlo simulations for a system of 1000 molecules, with runs of at least 160000 cycles for each state point.

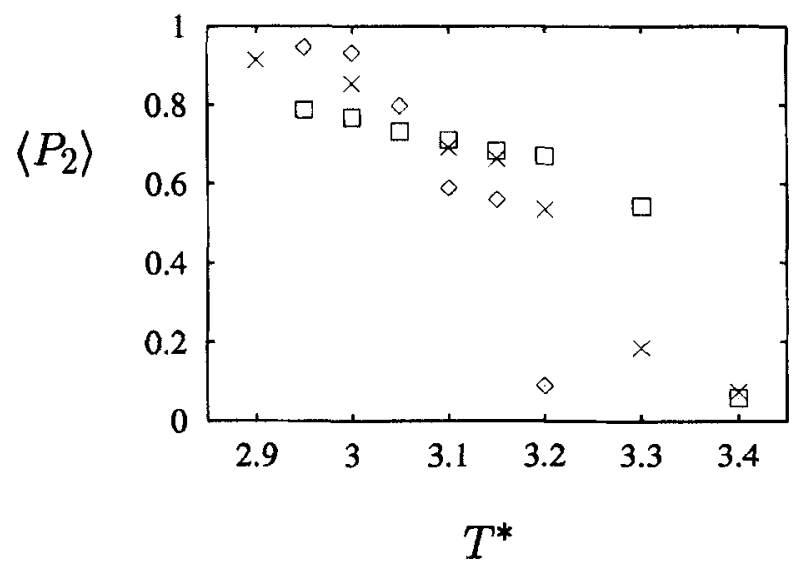

FIGURE 1: Scalar order parameter $\left\langle P_{2}\right\rangle$ vs. temperature $T^{*}$. Molecular steric dipole $p^{*}=0.814$ (diamonds), $p^{*}=0.662\left(\mathrm{X}\right.$-symbols), $p^{*}=0.524$ (squares).

When analyzing the simulation data we have extracted the quantities necessary to evaluate flexoelectric coefficients according to (7) and (8). Scalar nematic order parameters are directly accessible from a diagonalization of the molecular alignment tensor. The DPCF, 
$c(\mathbf{i}, \mathbf{j})$, is related to the total pair correlation function (TPCF), $h(\mathbf{i}, \mathbf{j})$, which accounts for spatial correlations of pairs of molecules in the system. (Here $\mathbf{i}$ denotes both position and orientation of molecule $i$ ) The conversion from the TPCF to the DPCF is established by the Ornstein-Zernike equation, which is an convolution integral equation,

$$
h(\mathbf{i}, \mathbf{j})=c(\mathbf{i}, \mathbf{j})+\rho \int \mathrm{d}(\mathbf{k}) f(\mathbf{k}) c(\mathbf{i}, \mathbf{k}) h(\mathbf{k}, \mathbf{j}) .
$$

The Ornstein-Zernike equation can be solved iteratively for the DPCF in an isotropic approximation,i.e., with $f(\mathbf{k})=\frac{1}{4 \pi}$, by applying a factorization technique according to Blum ${ }^{[6]}$. This method and its limitations are discussed in detail in Refs. ${ }^{[7-9]}$.

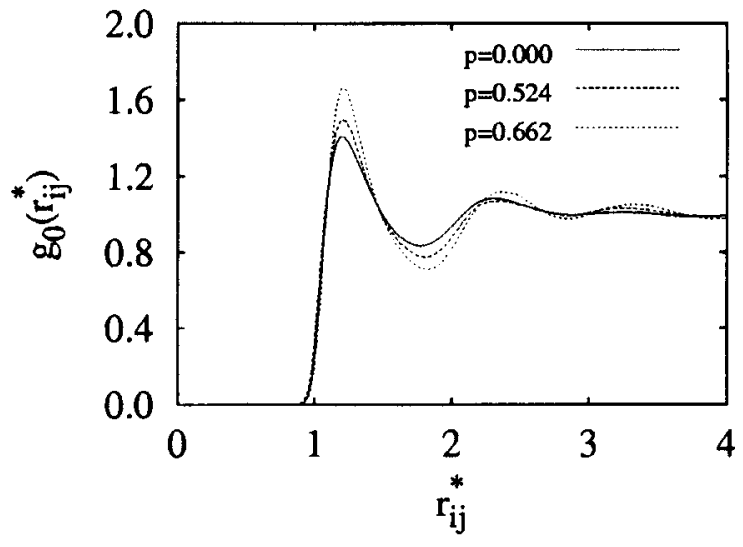

FIGURE 2: Radial correlation function $g_{0}\left(r_{i j}\right)$ between the ellipsoid centers for state points with $\left\langle P_{2}\right\rangle=0.69 \pm$ 0.01 . The continuous line is for $p^{*}=0$, the dashed line is for $p^{*}=0.524$ and the dotted line is for $p^{*}=0.662$.

\section{RESULTS}

Figure 1 reports the temperature dependence of the scalar order parameter, $\left\langle P_{2}\right\rangle$, for the three model systems with different steric dipoles. We found $\left\langle P_{1}\right\rangle=0$ within resolution of our computer experiments in all cases studied. We find that the effect of the spherical site is to reduce or even suppress the temperature stability range of the nematic phase in favor of the smectic phase. For $p^{*}=0.814$ 
there is a sharp phase transition from the smectic $A$ directly to the isotropic phase. (Smectic order has been detected from axial pair correlations.) With decreasing steric dipole the phase transition becomes smoother. For $p^{*}=0.662$ a nematic phase emerges between temperatures $T^{*}=3.1$ and 3.4. The nematic range becomes broader for $p^{*}=0.524$, lying between $T^{*}=2.95$ and 3.4. This range is still much smaller than for the pure Gay-Berne system where it spans the range $T^{*}=2.5$ and 3.2 at pressure $P^{*}=8$. (All quantities were measured in dimensionless units, referred to energy and length unit of the Gay-Berne model ${ }^{[4]}$.)

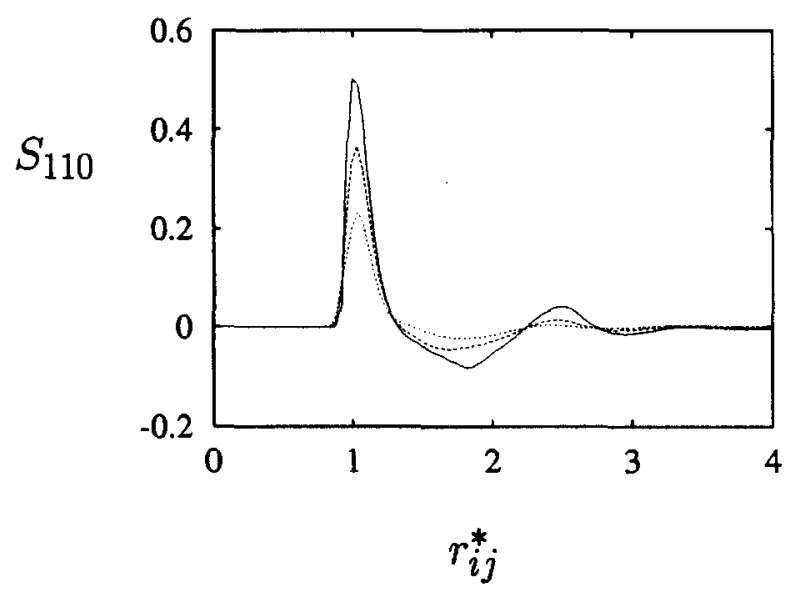

FIGURE 3: Rotational invariant $S_{110}$ vs. molecular separation $r_{i j}^{*}$ for molecular steric dipole $p^{*}=0.662$, and $T^{*}=$ 3.0 (solid), $T^{*}=3.2$ (dashed), $T^{*}=3.4$ (dotted).

The structure of the nematic is quite similar to that of the symmetric GB as we can see from the radial distribution $g_{0}\left(r_{i j}\right)$ in Figure 2. This shows the distribution of the ellipsoid centers

$$
g_{0}\left(r_{i j}\right)=\frac{1}{4 \pi r_{i j}^{2} \rho}\left\langle\delta\left(r_{i j}-r_{12}\right)\right\rangle_{12},
$$

calculated for the state points with $\left\langle P_{2}\right\rangle=0.69 \pm 0.01$ for the three systems: (a) $p^{*}=0$ at $T^{*}=3.30, \rho^{*}=0.30,\left\langle P^{*}\right\rangle=9,\left\langle U^{*}\right\rangle=-5.4$, $\left\langle P_{2}\right\rangle=0.68$; (b) $p^{*}=0.524$ at $T^{*}=3.15,\left\langle\rho^{*}\right\rangle=0.28, P^{*}=10$, $\left\langle U^{*}\right\rangle=-11.2,\left\langle P_{2}\right\rangle=0.68 ;$ and $(\mathrm{c}) p^{*}=0.662$ at $T^{*}=3.10,\left\langle\rho^{*}\right\rangle=$ 
$0.27, P^{*}=10,\left\langle U^{*}\right\rangle=-12.1,\left\langle P_{2}\right\rangle=0.69$. We can see that these curves are very similar and thus that the introduction of the steric dipole has brought a limited structural alteration.

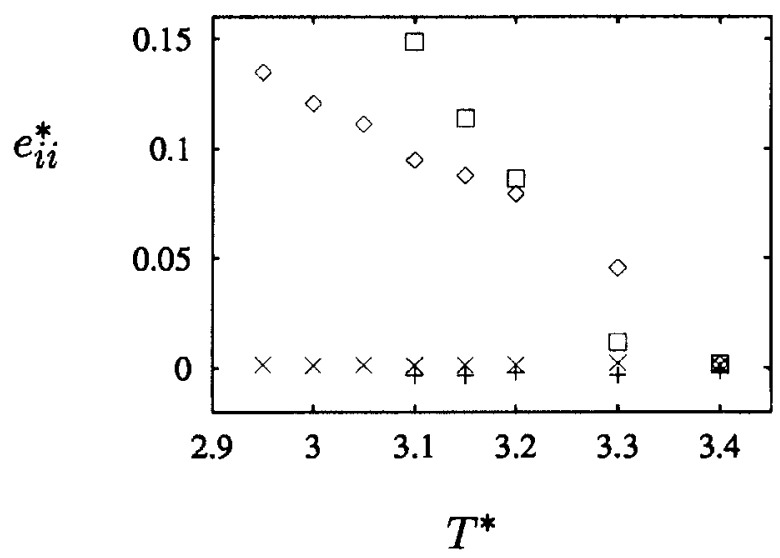

FIGURE 4: Flexoelectric coefficients $e_{i j}^{*}$ vs. temperature $T^{*}$. Diamonds: $e_{11}^{*}\left(p^{*}=0.524\right), \times$-symbols: $e_{33}^{*}$ $\left(p^{*}=0.524\right)$, squares: $e_{11}^{*}\left(p^{*}=0.662\right)$, crosses: $e_{33}^{*}\left(p^{*}=0.662\right)$.

In order to study spatial correlations of the molecular orientations we examined some of the averaged Stone functions ${ }^{[10]}$. In particular, the rotational invariant $S_{110}$, with

$$
S_{110}\left(r_{i j}\right)=-\frac{1}{\sqrt{3} \rho}\left\langle\delta\left(r_{i j}-r_{12}\right)\left(\mathbf{u}_{1} \cdot \mathbf{u}_{2}\right)\right\rangle_{12},
$$

provides information on the average orientation between the axes of two molecules at distance $r_{12}$. In Figure 3 we plot this function, for steric dipole $p^{*}=0.662$, at temperatures $T^{*}=3.0,3.2,3.4$, corresponding to the smectic $A$, nematic and isotropic phase, respectively. Two features are important. The first is that, the largest peak in Figure 3 is positive, which means that nearest neighbors tend to align antiparallel. Secondly, the long range asymptotic limit of $S_{110}$, which formally is proportional to the scalar order parameter $\left\langle P_{1}\right\rangle$, reaches almost zero. Both from the local antiparallel arrangement of the steric dipoles and from the vanishing odd rank scalar order parameters we can deduce that the formation of a macroscopic polarization 
is not present. Therefore, flexoelectric effects in our model systems should be expected to be rather small.

This is confirmed from the numerical evaluation of the flexoebctric coefficients according to (7) and (8). Especially, the smallness of $e_{33}^{*}$, which falls within the errorbars of our calculations, suggests that there is hardly any bend flexoelectric effect. Indeed, Meyer had already pointed out that for pear-shaped molecules the polarization couples to a splay distortion, whereas a banana shape of the molecules should be necessary in order to produce a bend flexoelectric effect ${ }^{[1]}$. When we compare the two systems it is obvious that a more pronounced molecular steric dipole leads to a considerable increase of the splay flexoelectric coefficient $e_{11}^{*}$ (Figure 4).

To summarize, we have shown that flexoelectric coefficients can be obtained by computer simulations, starting from a simple molecular model which allows for controlled asymmetric deformations.

\section{ACKNOWLEDGMENTS}

We gratefully acknowledge support from CNR, MURST (PRIN Cristalli Liquidi), University of Bologna and the European Union (TMRFMRX CT970121) and, in particular, a M. Curie TMR grant to J. Stelzer (TMR-ERB4001GT970528).

\section{References}

[1] R. B. Meyer, Phys. Rev. Lett. 22, 918 (1969).

[2] J. P. Straley, Phys. Rev. A 14, 1835 (1976).

[3] J. M. Haile, C. G. Gray, Chem. Phys. Lett. 76, 583 (1980)

[4] J. G. Gay, B. J. Berne, J. Chem. Phys. 74, 3316 (1981).

[5] R. Berardi, C. Fava, C. Zannoni, Chem. Phys. Lett. 297, 8 (1998).

[6] L. B]um, J. Chem. Phys. 58, 3295 (1973).

[7] J. Stelzer, C. Mason, E. de Miguel, M. P. Allen, Phys. Rev. E 52, R25 (1995).

[8] J. Stelzer, L. Longa, H.-R. Trebin, J. Chem. Phys. 103, 3098 (1995); 107, 1295 E (1997).

[9] J. Stelzer, R. Berardi, C. Zannoni, Chem. Phys. Lett. 299, 9 (1999).

[10] A. J. Stone, Mol. Phys. 36, 241 (1978). 